\title{
Intestinal perforation after radical cystectomy due to drain: Case report
}

\author{
Salih Budak ${ }^{1}$, Hüseyin Aydemir ${ }^{2}$, Hasan Salih Saglam ${ }^{2}$, Oztuğ Adsan ${ }^{2}$ \\ ${ }^{1}$ Tepecik Training and Research Hospital, Urology Clinic, Izmir, Turkey; \\ ${ }^{2}$ Sakarya University Training and Research Hospital, Urology Clinic, Sakarya, Turkey
}

\begin{abstract}
Summary The current standard treatment for nonmetastatic invasive bladder cancer is radical cystectomy with urinary diversion. Radical cystectomy surgery carries a serious potential risk of complications. In this case report, an intestinal perforation which was thought to be occurred due to a Foley catheter placed as a drain after the cystectomy is presented.
\end{abstract}

KEY WORDS: Cystectomy; Intestinal perforation; Drainage.

Submitted 28 July 2014; Accepted 30 September 2014

\section{INTRODUCTION}

The current standard treatment for the non-metastatic invasive bladder cancer is radical cystectomy with urinary diversion. Despite of the technical improvements, nowadays, radical cystectomy surgery carries a serious potential risk of complications. Although the improvements in the perioperative and postoperative care decrease the complication risks in years, the mortality rate of cystectomy is still 3\% and the post-surgical morbidity rate is $30 \%$ (1).

Cystectomy with urinary diversion is a serious surgical procedure for the physiological reserve.

In this article, we presented a case of intestinal (ileum) perforation complicating the postoperative period of a patient who underwent radical cystectomy and urinary diversion.

\section{Case report}

A sixty-five year-old female patient presented with a recurrent macroscopic hematuria complaint. It was learned from her history that the patient was a smoker at a rate of 40 packets/year and that she did not have any other disease. At initial evaluation, there was no abnormal finding at physical examination and serum biochemical and hematological investigations were in the normal range. At ultrasonography of the urinary tract, both kidneys were normal but an approximately $4-5 \mathrm{~cm}$ large mass lesion was detected in the anterior wall of the bladder.

A complete resection of the tumor formation, which was approximately $5 \mathrm{~cm}$ in size on the anterior wall of the bladder, was performed. The histopathological evaluation report of the surgical material demonstrated a muscle invasive urethral carcinoma (T2G3). A radical cystectomy with urinary diversion by an ileal reservoir was proposed to the patient.

Radical cystectomy, bilateral pelvic lymphadenectomy and ileal conduit diversion were performed. Urethra, uterus, cervix, ovaries and anterior wall of the vagina were removed with the bladder due to the involvement of the bladder neck. Bricker technique was applied for uretero-ileal anastomosis. As an abnormal finding during the surgery, there was a tumor extension from the bladder anterior wall to the symphysis pubis. At the end of the operation, a silicon drain was placed into the abdomen and a Foley balloon catheter was placed into the urethrectomy region as drainage.

The patient was mobilized in the postoperative 1st day and the nasogastric tube was removed.

The oral feeding (regime 1) started in the postoperative third day, total parenteral nutrition was discontinued and the vaginal tampon was removed. Defecation occurred in the postoperative forth day and regime 2 was started, the abdominal drain was removed, but the Foley drain was left in place because it drained $300 \mathrm{cc}$. The deep vein thrombosis prophylaxis was ended in the postoperative fifth day and there was stool outflow. In the fifth postoperative day nausea, abdominal pain and sensitivity occurred with increased white blood cell count. Oral nutrition was stopped and intravenous liquid replacement was started.

A general surgery consultation was requested. The patient was followed with blood count evaluations, direct abdominal radiograph and findings of abdominal examinations. At the abdominal CT (with oral contrast) presence of free contrast between the intestinal loops was shown.

The patient was surgically explored in the postoperative fifth day.

At surgical exploration at approximately $20 \mathrm{~cm}$ apart from the ileocecal junction, the $15 \mathrm{~cm}$ long ileum wall including the intestinal anastomosis line was thickened and there was a perforation of both the proximal and the distal ileum with respect to the anastomosis, although the anastomotic line was still intact (Figure 1). 


\section{Figure 1.}

White arrow: distal perforation,

Green arrow: anastomotic line, Blue arrow: proximal perforation, Yellow arrow: ileal loop orifice.

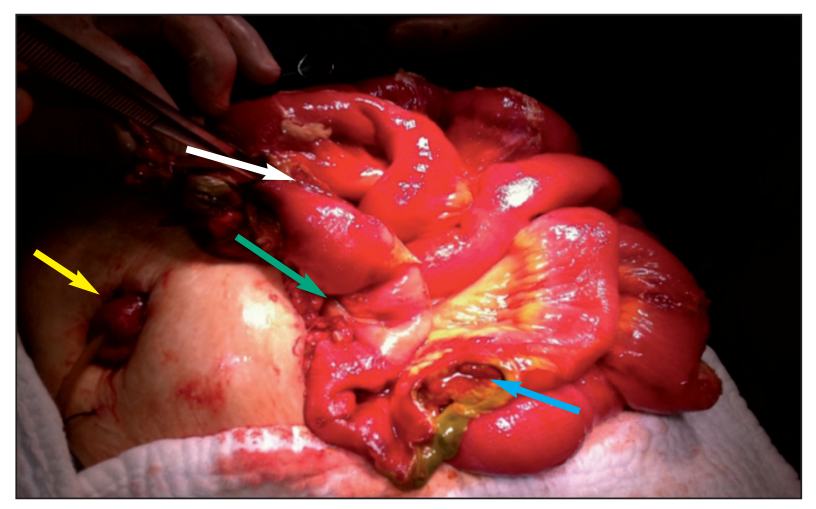

\section{Figure 2.}

Exploration finding, two perforations in the ileum segment, White arrow: distal perforation,

Green arrow: anastomose line,

Blue arrow: proximal perforation.

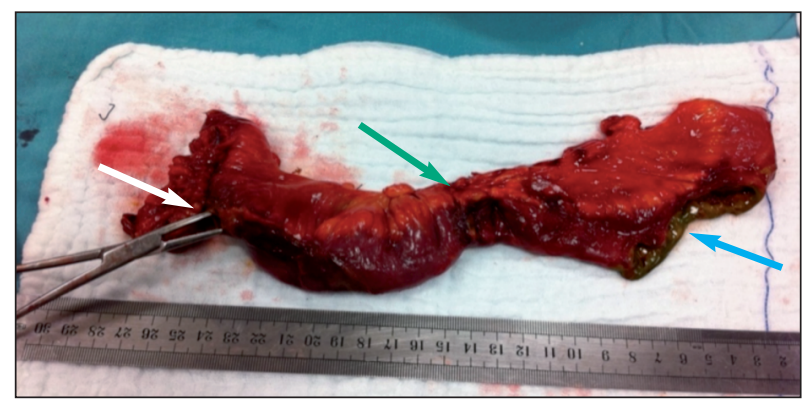

İleum resection was performed in a way to include the two perforation areas and the anastomotic line (Figure 2). Illeostomy was anastomosed to the skin.

\section{Discussion}

In the cases of bladder tumors invasive to the muscle, cystectomy is the most effective treatment choice with a disease-free survival rate of $87 \%$ (2). Postoperative close follow-up is important for the diagnosis and treatment of early complications. Since cystectomy and urinary diversion operations are serious operation for the physiological functions, the hospitalization periods of the patients might be approximately more than one week. Complications of the early period after radical cystectomy occurs approximately at a rate of 6-30\% and consist mostly in bleeding, intestinal obstruction, urinary extravasation, urinary reflux and infection (1). In our clinics, we restore oral nutrition and mobilization in the patients who underwent cystectomy in our clinics as soon as possible after the operation. The intestinal epithelium is quite sensitive to anoxia and necrosis and they can easily occur in the areas of the operation resulting in perforation. Intestinal perforation may occur as a result of pressure or ischemic necrosis (3). Although the early period of follow-up after the operation was in the normal course in our case, we think that the Foley drain that we placed into the urethrectomy

\section{Figure 3.}

Blue arrow: proximal perforation, Green Arrow: urethrectomy region drain (Foley catheter).

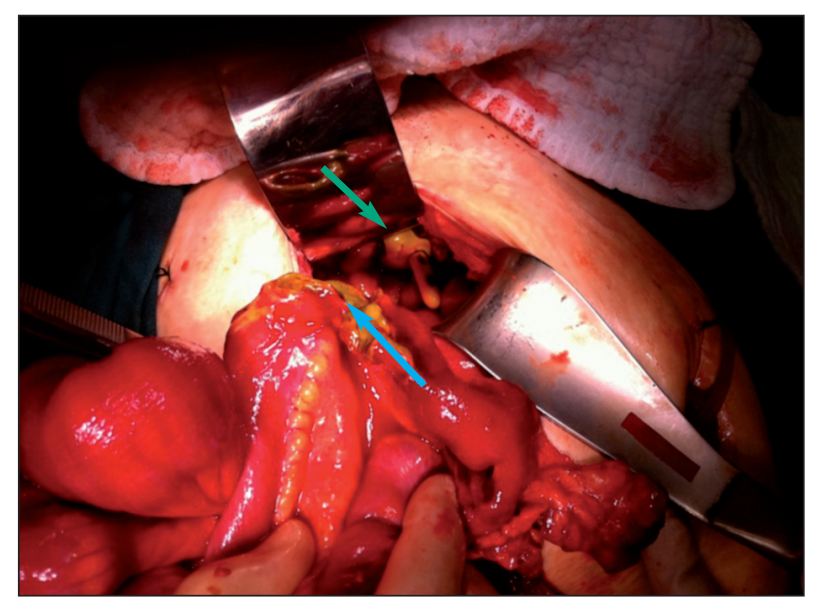

region might have led to the necrosis of the ileum wall by making a physical pressure. As can be understood from Figure 3, erosion developed in the areas that had a contact with the foreign body.

In conclusion, it should be taken into account that in the patients who had urinary diversion by using intestinal segments, the fragility of the intestinal structures increase. Thus, one should be careful in the placement of stents, drains and catheters. The early follow-up after the radical cystectomy should be carefully and drains and catheters should be removed as soon as possible.

\section{REFERENCES}

1. Maffezzini M, Gerbi G, Campodonico F, Parodi D. Multimodal perioperative plan for radical cystectomy and intestinal urinary diversion. Effect on recovery of intestinal function and occurrence of complications. Urology. 2007; 69:1107-1111.

2. Stein JP, Leiskowsky G, Cote R, et al. Radical cystectomy in treatment of invasive bladder cancer: long-term results in 1,054 patients. J Clin Oncol. 2002; 19: 666-675.

3. Kulaylat MN, Doerr RF. Small bowel obstruction. Holzhimer RG, Mannick JA (eds) Surgical Treatment: Evidence-Based and ProblemOriented. 2001.

\section{Correspondence}

Salih Budak,MD (Corresponding Author) salihbudak1977@gmail.com

Tepecik Training and Research Hospital, Urology Clinic, Izmir, Turkey

Hüseyin Aydemir, MD

husaydemir@yahoo.com

Hasan Salih Saglam, MD Associate. Prof.

hasanss@hotmail.com

Oztuiğ Adsan, MD Professor oztugadsan@yahoo.com

Sakarya EAH, Uroloji Kliniği, Sakarya, Turkey 